\title{
Linear evasion differential game of one evader and several pursuers with integral constraints
}

\author{
Gafurjan Ibragimov ${ }^{1,2} \cdot$ Massimiliano Ferrara $^{2,3}$ (D) Marks Ruziboev ${ }^{4}$. \\ Bruno Antonio Pansera ${ }^{2}$
}

Accepted: 24 January 2021 / Published online: 11 February 2021

(c) The Author(s) 2021, corrected publication 2021

\begin{abstract}
An evasion differential game of one evader and many pursuers is studied. The dynamics of state variables $x_{1}, \ldots, x_{m}$ are described by linear differential equations. The control functions of players are subjected to integral constraints. If $x_{i}(t) \neq 0$ for all $i \in$ $\{1, \ldots, m\}$ and $t \geq 0$, then we say that evasion is possible. It is assumed that the total energy of pursuers doesn't exceed the energy of evader. We construct an evasion strategy and prove that for any positive integer $m$ evasion is possible.
\end{abstract}

Keywords Evasion differential game · Evader · Many Pursuers · Integral constraint · Evasion $\cdot$ Strategy

The original article the OA funding note was missing.

\assimiliano Ferrara

massimiliano.ferrara@unirc.it; massimiliano.ferrara@unibocconi.it

Gafurjan Ibragimov

ibragimov@upm.edu.my; gafurjan.ibragimov@unirc.it

Marks Ruziboev

marx.ruziboev@gmail.com

Bruno Antonio Pansera

bruno.pansera@unirc.it

1 Department of Mathematics and Institute for Mathematical Research, Universiti Putra Malaysia, Seri Kembangan, Malaysia

2 Department of Law, Economics and Human Sciences, University Mediterranea of Reggio Calabria, Reggio Calabria, Italy

3 Department of Management and Technology, ICRIOS-The Invernizzi Centre for Research in Innovation, Organization, Strategy and Entrepreneurship Bocconi University, Milan, Italy

4 Mathematics Institute, Leiden University, Leiden, The Netherlands 


\section{Introduction}

The field of differential games was pioneered by Isaacs (1965) in the 1960s and since then enormous amount of work has been devoted to its study (for example, see Friedman 1971; Hajek 1975; Pontryagin 1988; Krasovskii and Subbotin 1988; Petrosyan 1993; Başar and Olsder 1999; Buckdahn et al. 2011 and references therein). A substantial part of these works concern simple motion pursuit and evasion differential games of many players. Often, either geometric or integral constraints are imposed on the control parameters of players. In Croft (1964) Croft showed that in the $n$-dimensional Euclidean ball $n$ lions can catch the man, while the man can escape from $n-1$ lions when the controls of the players are subject to geometric constraints. A similar game problem was studied by Ivanov (1980) on any convex compact set and an estimate from above was obtained for guaranteed pursuit time.

Interesting results were obtained in Alexander et al. (2009), Azamov (2008), Bakolas and Tsiotras (2011), Berkovitz (1986), Bhattacharya et al. (2016), Konstantinidis and Kehagias (2016) and Sun and Tsiotras (2014) for various games in unbounded regions as well as on graphs. Evasion problem on time interval $\left[t_{0}, \infty\right)$ was introduced and studied by Pontryagin and Mischenko (1971). In Mishchenko et al. (1977) Mishchenko et al. proposed a new manoeuvre for evasion in the game of many pursuers.

Chernous'ko (1976) studied an evasion game of one evader and several pursuers with a state constraint, i.e. the evader was supposed to remain in a neighborhood of a given ray for the duration of the game. He proved that if the evader is faster than the pursuers then evasion is possible. This result was extended by Chernous'ko and Zak (1985) and Zak (1978, 1981, 1982) to more general differential game problems. Related problems of evasion from a group of pursuers were studied in Borowko et al. (1988) and Chodun (1989).

In Pshenichnii (1976) Pshenichnii considered a simple motion differential game of many pursuers and one evader in $\mathbb{R}^{n}$, when all players have the same dynamic possibilities. He proved that if the initial state of the evader belongs to the interior of convex hull of pursuers' initial states, then pursuit can be completed, otherwise evasion is possible. Based on this work, Pshenichnii et al. (1981) developed the method of resolving functions for solving linear pursuit problems with many pursuers. Later on, the results of paper (Pshenichnii 1976) were extended by many researchers to cover various cases. For example, when control sets of players are convex compact sets, Grigorenko (1990) obtained the necessary and sufficient conditions of evasion of one evader from several pursuers.

The papers Chikrii and Prokopovich (1992) and Kuang (1986) are also extensions of Pshenichnii (1976). In Kuchkarov et al. (2012) the game problem of many pursuers and one evader was studied on a cylinder. In the recent work of Kuchkarov et al. (2016), the results of Pshenichnii (1976) were extended to differential games on manifolds with Euclidean metric. In Blagodatskikh and Petrov (2009) Blagodatskikh and Petrov obtained necessary and sufficient condition of evasion in a simple motion differential game of a group of pursuers and a group of evaders in $\mathbb{R}^{n}$ where all evaders use the same control. By definition, pursuit is considered completed if the state of a pursuer coincides with the state of at least one evader. Also, the works (Bannikov and Petrov 
2010; Vagin and Petrov 2001) related to such games. Recently in Scott and Leonard (2018) the authors consider a pursuit-evasion game involving one pursuer and multiple evaders motivated by the seminal "selfish herd" model of Hamilton (1971). The pursuer can freely move in any direction with bounded speed and evaders move with bounded speed and bounded turning speed. Using Isaacs' heuristic argument they constructed an optimal strategy for the pursuer and concluded that the optimal strategy for the pursuer is to focus on a single evader that can be captured in minimum time. Moreover, "nontargeted" evaders are always able to escape. We refer to Kumkov et al. (2017) for a survey of results on differential games of many players with geometric constraints. In the case of integral constraints, simple motion evasion games of many players were solved in Alias et al. (2016), Ibragimov et al. (2012) and Ibragimov et al. (2018).

In the present paper, we study a linear evasion differential game of many pursuers and one evader. The controls of players are subjected to integral constraints. To the best of our knowledge no previous study has investigated the linear evasion game problem stated in the present paper. The main difficulties in solving the problem are the construction of evasion strategy and to prove the fact that the objects go around the origin on some specified time interval $[0, T]$ maintaining some distances from the origin. Note that we employ non-anticipative strategies in the present game model (see, for example, Cardaliaguet et al. 2000).

Note that there is a similarity between the constructed evasion strategies and proofs of the main results of current and existing works (Ibragimov et al. 2012, 2018). They are (i) the definition of time intervals $\left[\tau_{i}, \tau_{i}^{\prime}\right.$ ), (ii) the construction of a strategy for the evader which allows the evader to use a manoeuvre on $\left[\tau_{i}, \tau_{i}^{\prime}\right)$ against the $i$-th pursuer, (iii) estimating the distances between the evader and pursuers, and establishing that evasion is possible. We believe that these steps will be common for the most of open evasion differential game problems of many players with integral constraints described by the linear system of equations as well. However, the main difficulties in solving the open problems will remain to overcome the steps (i)-(iii) listed above.

In the case of linear equations studied in the present paper, the strategies of existing papers do not work since, for the linear equation describing the game, we have to find its own $\tau_{i}$. Also, by contrast, we need bounded $\tau_{i}, \tau_{i}^{\prime}$ and new techniques to estimate $x_{p}(t)$. Note that according to the strategy of the present paper, in contrast to previous works, each object, for any control functions of pursuers, moves with a positive speed in the direction of $y$-axis on the time interval $[0, T]$. Moreover, all the objects will become on the upper half plane by the time $T$, and then evasion is established. The fact that each time interval where the evader uses a manoeuver is contained in the interval $[0, T]$ plays a key role in establishing a number of estimates in the proof of the main result.

This work is a milestone study to undertake a detailed analysis of linear evasion differential game problem of many pursuers and one evader with integral constraints. We are confident that the construction in the present paper will be a stepping stone to open problems and will open prospects for general multi person linear evasion differential games with integral constraints and this study makes a major contribution to research on general linear evasion differential games. 


\section{Statement of problem}

Let $x_{1}, \ldots, x_{m}, m \geq 1$, be the points moving in $\mathbb{R}^{n}$ whose dynamics are described by the equations

$$
\dot{x}_{i}=-\lambda_{i} x_{i}+v-u_{i}, \quad x_{i}(0)=x_{i}^{0}, \quad i=1,2, \ldots, m,
$$

where $u_{1}, \ldots, u_{m}$ are the control parameters of pursuers and $v$ is that of evader, $\lambda_{i}>0$, $x_{i}, x_{i}^{0}, u_{i}, v \in \mathbb{R}^{n}, n \geq 2, x_{i}^{0} \neq 0, i=1, \ldots, m$.

Definition 2.1 Measurable functions $u_{i}(t)$ and $v(t), t \geq 0$, that satisfy the following integral constraints

$$
\int_{0}^{\infty}\left|u_{i}(t)\right|^{2} d t \leq \rho_{i}^{2}, i=1, \ldots m ; \quad \int_{0}^{\infty}|v(t)|^{2} d t \leq \sigma^{2}
$$

are called controls of the $i$ th pursuer and evader, respectively.

Definition 2.2 A function $\left(t, t_{1}, \ldots, t_{k}, x_{1}, \ldots, x_{m}, u_{1}, \ldots, u_{m}\right) \mapsto V\left(t, t_{1}\right.$, $\left.\ldots, t_{k}, x_{1}, \ldots, x_{m}, u_{1}, \ldots, u_{m}\right), V:[0, \infty)^{k+1} \times \mathbb{R}^{2 n m} \rightarrow \mathbb{R}^{n}$, where $t_{1}, \ldots, t_{k}$, $0<t_{1}<\cdots<t_{k}<\infty$, are some positive numbers (unspecified) and $k$ is a positive integer, is called strategy of evader if the following system of equations

$$
\begin{aligned}
& \dot{x}_{i}=-\lambda_{i} x_{i}+V\left(t, t_{1}, \ldots, t_{k}, x_{1}, \ldots, x_{m}, u_{1}, \ldots, u_{m}\right)-u_{i}, \\
& x_{i}(0)=x_{i}^{0}, i=1, \ldots, m,
\end{aligned}
$$

has a unique solution $\left(x_{1}(t), \ldots, x_{m}(t)\right), t \geq 0$, for any controls $\left(u_{1}(t), \ldots, u_{m}(t)\right)$, of pursuers and along this solution

$$
\int_{0}^{\infty}\left|V\left(t, t_{1}, \ldots, t_{k}, x_{1}(t), \ldots x_{m}(t), u_{1}(t), \ldots, u_{m}(t)\right)\right|^{2} d t \leq \sigma^{2} .
$$

The strategy $V\left(t, t_{1}, \ldots, t_{k}, x_{1}, \ldots, x_{m}, u_{1}, \ldots, u_{m}\right)$ is nonanticipatively defined with respect to the strictly increasing finite sequence of numbers $t_{1}, \ldots, t_{k}$ as follows. Let the time $t_{i}\left(t_{0}=0\right), i=0,1, \ldots, k$, be occurred. The strategy of the evader is defined on the time interval $\left[t_{i}, t_{i+1}\right), i=0,1, \ldots, k$, where $t_{k+1}=+\infty$, as a function $V=V^{i}\left(t, t_{1}, \ldots, t_{i}, x_{1}, \ldots, x_{m}, u_{1}, \ldots, u_{m}\right)$. The trajectories of objects $x_{1}(t), \ldots, x_{m}(t)$ i.e. the solution of (3) generated by this strategy and arbitrary controls of pursuers $u_{1}(t), \ldots, u_{m}(t)$ are then defined as the solution of the initial value problem

$$
\begin{aligned}
& \dot{x}_{j}=-\lambda_{j} x_{j}+V^{i}\left(t, t_{1}, \ldots, t_{i}, x_{1}, \ldots, x_{m}, u_{1}, \ldots, u_{m}\right)-u_{j},\left.\quad x_{j}(t)\right|_{t=t_{i}} \\
& \quad=x_{j}\left(t_{i}\right), j=1, \ldots, m
\end{aligned}
$$

until the time $t_{i+1}, i=0,1, \ldots, k$, occurs. The number $t_{i+1}$ is defined as the first time when the points $x_{1}(t), \ldots, x_{m}(t)$ satisfy a certain condition. In this way, we define 
the solution of (3) on the intervals $\left[t_{i}, t_{i+1}\right), j=0,1, \ldots, k$. It should be noted that the evader can predict neither the values of $t_{1}, \ldots, t_{k}$ nor the length of the interval $\left[t_{i}, t_{i+1}\right), i=0,1, \ldots,(k-1)$.

Definition 2.3 If there exists a strategy $V$ of evader such that for any controls of pursuers $x_{i}(t) \neq 0, i=1, \ldots, m, t \geq 0$, then we say that evasion is possible.

The problem is to find a condition for evasion to be possible.

Thus, the evader knows the values $x_{1}(t), \ldots, x_{m}(t), u_{1}(t), \ldots, u_{m}(t)$ of parameters $x_{1}, \ldots, x_{m}, u_{1}, \ldots, u_{m}$ at the current time $t$. Pursuers apply arbitrary controls $u_{1}(t), \ldots, u_{m}(t), t \geq 0$, and try to realize the equation $x_{i}(t)=0$ at least for one $i \in\{1,2, \ldots, m\}$, whereas the evader tries to maintain the inequalities $x_{i}(t) \neq 0$ for all $i=1, \ldots, m$ and $t \geq 0$.

\section{The main result}

In this section we prove a theorem about evasion. To this end, we specify the conditions to define the numbers $t_{i}$ and construct an explicit nonanticipative strategy for the evader.The following is the main result of the current paper.

Theorem 3.1 If

$$
\rho_{1}^{2}+\cdots+\rho_{m}^{2} \leq \sigma^{2}
$$

then evasion is possible in game (1)-(2).

We prove the theorem in several subsections. The proof strategy is as follows. The solution of the initial value problem (1) is given by

$$
x_{i}(t)=e^{-\lambda_{i} t} y_{i}(t), \quad y_{i}(t)=x_{i}^{0}+\int_{0}^{t} e^{\lambda_{i} s}\left(v(s)-u_{i}(s)\right) d s .
$$

Since $x_{i}(t)=0$ if and only if $y_{i}(t)=0$, below we study the evolution of $y_{i}(t)$. We construct an evasion strategy such that the second coordinate of each point $y_{i}(t)$ strictly increases all the time. It remains to look at the situation with initial state $y_{i}(0)$ with $y_{i 2}(0)<0$ for some $i$.

Define an $a_{i}$-approach time $t=\tau_{i}$ as the first time for which $\left|y_{i}(t)\right|=a_{i}$ and $y_{i 2}(t)<0$. $\left(a_{i}\right)$ is a strictly decreasing sequence so $\tau_{i}$ (if ever defined) is increasing. Time $\tau_{i}^{\prime}>\tau_{i}$ is specified to ensure that $y_{i 2}\left(\tau_{i}^{\prime}\right)>0$ if a "manoeuvre" is deployed on the time interval $\left[\tau_{i}, \tau_{i}^{\prime}\right)$.

More essential for the proof of the theorem is that the sequence $\left\{\tau_{i}\right\}$ is bounded such that the set $I_{1}=\bigcup_{i=1}^{m_{0}}\left[\tau_{i}, \tau_{i}^{\prime}\right)$ is contained in $[0, T]$ for some $T$. This is essentially implied by the fact that $y_{i 2}(t)$ is strictly increasing for all $i$ and at any time $t>0$, and the definition for the $a_{i}$-approach time $\tau_{i}$ requires $y_{i 2}(t)<0$.

The "manoeuvre" is defined (see (16)) such that once some object $y_{i}\left(\tau_{p}\right)$ is close to the origin, i.e. $\left|y_{i}\left(\tau_{p}\right)\right|=a_{p}$ while $y_{i 2}\left(\tau_{p}\right)<0$ for some $\tau_{p}$, some energy is 
allocated on the first coordinate in order to increase $\left|y_{i 1}(t)\right|$ (such that $\left|y_{i}(t)\right| \geq a_{p+1}$ on $\left[\tau_{p}, \tau_{p}^{\prime}\right]$, (see (55)), avoiding the origin). Also, $y_{i 2}\left(\tau_{p}\right)$ increases on $\left[\tau_{p}, \tau_{p}^{\prime}\right]$ (such that $y_{i 2}(t) \geq a_{p}$ on $\left[\tau_{p}^{\prime}, \infty\right)$ (see (56))). The objective is that after the distance of point $y_{i}(t)$ from the origin is $a_{p}$ at $t=\tau_{p}$, it is not possible for it to be again at an $a_{q}$ distance at some $\tau_{q}$ with $q \geq p+1$ (see (53)-(55))

The auxiliary trajectory $z_{p}(t)$ is the trajectory of $y_{p}(t)$ if the evader applies the "manoeuvre" against the $p$-pursuer on the whole interval $\left[\tau_{p}, \tau_{p}^{\prime}\right)$. In the end, estimations of $\left|y_{p}(t)-z_{p}(t)\right|$ and $z_{p}(t)$ are needed to estimate $\left|y_{p}(t)\right|$.

\subsection{Notations}

It is sufficient to consider the case when $n=2$ and

$$
\rho^{2}:=\rho_{1}^{2}+\cdots+\rho_{m}^{2}<\sigma^{2}
$$

(see, for example, (Ibragimov et al. 2012, 2018).

Let $\alpha$ be any number satisfying the condition

$$
0<\alpha<\frac{(\sigma-\rho)^{2}}{2\left(\max _{1 \leq i \leq m}\left|x_{i}^{0}\right|+1\right)}
$$

We choose a number $a_{1}$ such that

$$
0<a_{1}<\min \left\{\frac{1}{2}, \frac{(\sigma-\rho)^{2}}{4 \alpha}, \frac{\sigma^{2}}{32 \alpha}, \frac{\alpha}{2 \Lambda}, \min _{i}\left|x_{i}^{0}\right|\right\}
$$

where $\Lambda=\max _{1 \leq i \leq m} \lambda_{i}$. Let

$T_{0}=\frac{1}{\alpha} \max _{1 \leq i \leq m}\left|x_{i}^{0}\right|, \quad T=T_{0}+\frac{2 a_{1}}{\alpha}, \quad \kappa=\min \left\{\frac{1}{2}, \frac{\alpha}{16 \sigma^{2} e^{\Lambda T}}, \frac{\alpha^{3}}{8 \cdot 6^{4} \sigma^{6} e^{4 \Lambda T}}\right\}$.

Let a sequence $\left\{a_{i}\right\}_{i=1}^{\infty}$ be defined by the formula $a_{i+1}=\kappa \cdot a_{i}^{4}$. It is not difficult to see that this sequence has the following

Property $3.2 \sum_{i=k}^{\infty} a_{i} \leq 2 a_{k}$ for any $k \geq 1$.

Let $y_{i}=\left(y_{i 1}, y_{i 2}\right), v=\left(v_{1}, v_{2}\right)$, and $u_{i}=\left(u_{i 1}, u_{i 2}\right)$. Define $a_{i}$-approach time $\tau_{i}$ to be the first time such that

$$
\left|y_{j}\left(\tau_{i}\right)\right|=a_{i}, \quad y_{j 2}\left(\tau_{i}\right)<0, \quad i=1, \ldots, m_{0},
$$

for some $j \in\{1, \ldots, m\}$, where $m_{0}$ is a positive integer. In Sect. 3.2 we'll show that $\tau_{i}$ are defined for some points $y_{i}, i=1, \ldots, m_{0}, m_{0} \leq m$. Note that $a_{i}$-approach times $\tau_{i}$ may not be defined as well $\left(m_{0}=0\right)$.

First we define $\tau_{1}$ if relations (10) are satisfied at $i=1$ for some $j$. Then, we define $\tau_{2}$ and so on. Therefore, $\tau_{1}<\tau_{2}<\cdots<\tau_{m_{0}}$. Note that times $\tau_{i}$ are unspecified and 
depend on the evader's strategy and the controls of the pursuers. It is important to note the fact that all the numbers $\tau_{i}$ will be in the interval $[0, T]$, which will be established in Sect. 3.2.

Without loss of generality we relabel $y_{j}$ for which $\left|y_{j}\left(\tau_{i}\right)\right|=a_{i}, y_{j 2}\left(\tau_{i}\right)<0$ by $y_{i}$. Note that the condition (10) can occur at the time $\tau_{i}$ for several $j$. If so, we label any one of them by $y_{i}$. Let

$$
\tau_{i}^{\prime}=\frac{1}{\lambda_{i}} \ln \left(e^{\lambda_{i} \tau_{i}}+\frac{2 \lambda_{i} a_{i}}{\alpha}\right), \quad i=1, \ldots, m_{0}
$$

Property 3.3 For any $i, k \in\left\{1, \ldots, m_{0}\right\}$,

(1) $\tau_{i}^{\prime}-\tau_{i} \leq \frac{2 a_{i}}{\alpha}$.

(2) $\sum_{i=k}^{m_{0}}\left(\tau_{i}^{\prime}-\tau_{i}\right) \leq \frac{4 a_{k}}{\alpha}$.

Proof To prove item (1), we have

$$
\tau_{i}^{\prime}-\tau_{i}=\frac{1}{\lambda_{i}} \ln \left(e^{\lambda_{i} \tau_{i}}+\frac{2 \lambda_{i} a_{i}}{\alpha}\right)-\tau_{i}
$$

Since for any $a \geq 1$ and $b \geq 0$ we have $\ln (a+b) \leq \ln a+b$, therefore (11) implies that

$$
\tau_{i}^{\prime}-\tau_{i} \leq \frac{1}{\lambda_{i}}\left(\lambda_{i} \tau_{i}+\frac{2 \lambda_{i} a_{i}}{\alpha}\right)-\tau_{i}=\frac{2 a_{i}}{\alpha} .
$$

The proof of item (2) follows from (12) as follows

$$
\sum_{i=k}^{m_{0}}\left(\tau_{i}^{\prime}-\tau_{i}\right) \leq \sum_{i=k}^{\infty} \frac{2 a_{i}}{\alpha}<\frac{4 a_{k}}{\alpha}
$$

using Property 3.2.

Further, we define a function $r:[0, \infty) \rightarrow\left\{0,1, \ldots, m_{0}\right\}$ as follows: set $r(t)=i$, if $t \in\left[\tau_{i}, \tau_{i}^{\prime}\right) \backslash I_{i+1}, i=0, \ldots, m_{0}$, where $\tau_{0}^{\prime}=\infty, I_{i}=\cup_{j=i}^{m_{0}}\left[\tau_{j}, \tau_{j}^{\prime}\right), I_{m_{0}+1}=\emptyset$. The function $r$ has the following useful property:

Property 3.4 For $i=1,2, \ldots,\left(m_{0}-1\right)$,

(1) $r(t)=i$ for $\tau_{i} \leq t<\tau_{i}^{\prime}$ if $\tau_{i}^{\prime} \leq \tau_{i+1}$.

(2) $r(t)=i$ for $\tau_{i} \leq t<\tau_{i+1}$ if $\tau_{i+1} \leq \tau_{i}^{\prime}$.

Proof Suppose that $\tau_{i}^{\prime} \leq \tau_{i+1}$. Then $\left[\tau_{i}, \tau_{i}^{\prime}\right) \backslash I_{i+1}=\left[\tau_{i}, \tau_{i}^{\prime}\right)$. Therefore, $r(t)=i$ for $t \in\left[\tau_{i}, \tau_{i}^{\prime}\right)$. This proves item (1).

To prove item (2), suppose that $\tau_{i+1} \leq \tau_{i}^{\prime}$. Since $\tau_{i}<\tau_{i+1}<\cdots<\tau_{m_{0}}$, we have $\left[\tau_{i}, \tau_{i+1}\right) \subset\left[\tau_{i}, \tau_{i}^{\prime}\right) \backslash I_{i+1}$. Therefore, $r(t)=i$ for $t \in\left[\tau_{i}, \tau_{i+1}\right)$ by definition. 


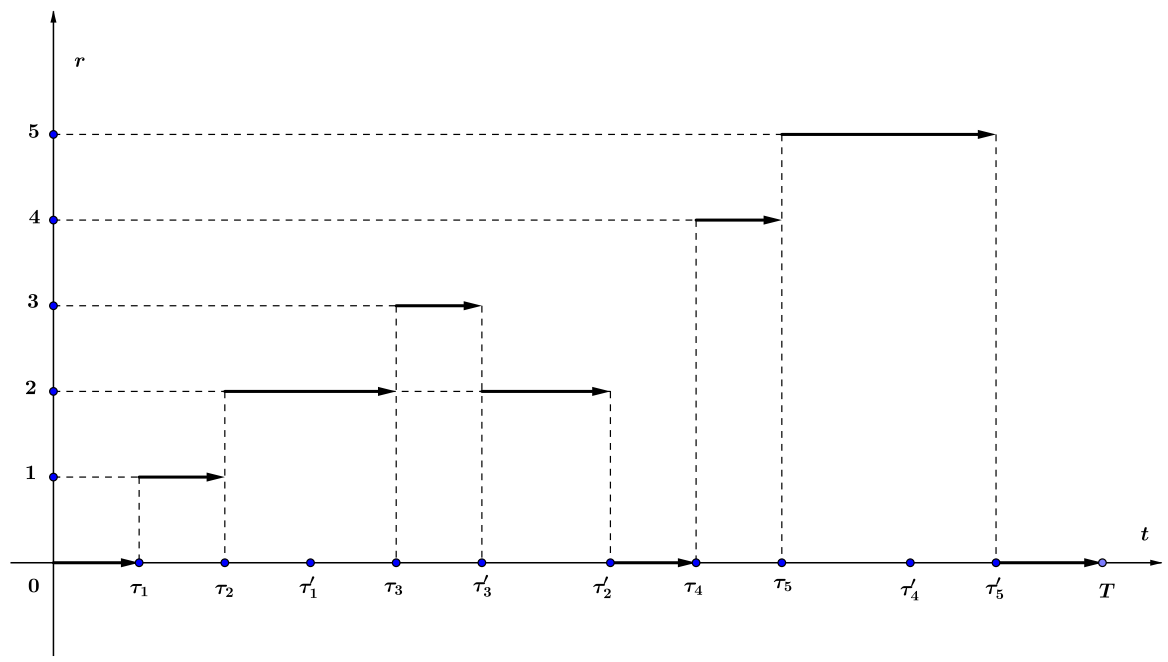

Fig. 1 The graph of function $r(x)$

\section{Example 3.5 If}

$$
0=\tau_{0}<\tau_{1}<\tau_{2}<\tau_{1}^{\prime}<\tau_{3}<\tau_{3}^{\prime}<\tau_{2}^{\prime}<\tau_{4}<\tau_{5}<\tau_{4}^{\prime}<\tau_{5}^{\prime},
$$

then $r(t)$ has the graph shown in Fig. 1 .

\subsection{Strategy for the evader}

Now we are ready to construct a strategy for the evader. Let $u_{j}(t), j=1, \ldots, m$, be arbitrary controls of pursuers. Set

$$
\begin{aligned}
& v(t)=V_{0}(t)=\left(0, \alpha+\left(\sum_{j=1}^{m}\left|u_{j}(t)\right|^{2}\right)^{1 / 2}\right), \quad t \in[0, T] \backslash I_{1}, \\
& v(t)=V_{r}(t)=\left(V_{r 1}(t), U(t)\right), t \in[0, T] \cap I_{1},
\end{aligned}
$$

where $r=r(t), V_{i}(t)=\left(V_{i 1}(t), U(t)\right), \tau_{i} \leq t<\tau_{i}^{\prime}, i=1, \ldots, m_{0}$, is defined as follows

$$
\begin{aligned}
V_{i 1}(t) & = \begin{cases}\alpha+\left|u_{i 1}(t)\right|, & y_{i 1}\left(\tau_{i}\right) \geq 0, \\
-\left(\alpha+\left|u_{i 1}(t)\right|\right), & y_{i 1}\left(\tau_{i}\right)<0,\end{cases} \\
U(t) & =\alpha+\left(\sum_{j=1}^{m} u_{j 2}^{2}(t)\right)^{1 / 2} .
\end{aligned}
$$


Note that $U(t)$ doesn't depend on $i$. Finally, let

$$
v(t)=\left(0,\left(\sum_{j=1}^{m}\left|u_{j}(t)\right|^{2}\right), \quad t>T .\right.
$$

Equation (15) shows that the function $r=r(t)$ assigns the control $V_{r}(t)$ for $v(t)$.

For example, if there are 5 pursuers and the numbers $\tau_{i}, \tau_{i}^{\prime}, i=1, \ldots, 5$, are arranged as in Example 3.5, then using the values of $r(t)$ in Fig. 1 we obtain from (15) that

$$
v(t)= \begin{cases}V_{1}(t) & \text { if } t \in\left[\tau_{1}, \tau_{2}\right), \\ V_{2}(t) & \text { if } t \in\left[\tau_{2}, \tau_{3}\right) \cup\left[\tau_{3}^{\prime}, \tau_{2}^{\prime}\right) \\ V_{3}(t) & \text { if } t \in\left[\tau_{3}, \tau_{3}^{\prime}\right), \\ V_{4}(t) & \text { if } t \in\left[\tau_{4}, \tau_{5}\right), \\ V_{5}(t) & \text { if } t \in\left[\tau_{5}, \tau_{5}^{\prime}\right) .\end{cases}
$$

On the intervals $\left[0, \tau_{1}\right),\left[\tau_{2}^{\prime}, \tau_{4}\right)$, and $\left[\tau_{5}^{\prime}, T\right]$, where $r(t)=0$ on $[0, T]$, the evader's strategy is defined by (14).

If $r(t)=i>0$ on some time interval, then we say that evader is applying a manoeuvre $V_{i}(t)$ against the $i$ th pursuer, or the evader is under the attack of the $i$-th pursuer on that interval. In Example 3.5, $r(t)=0$ on intervals $\left[0, \tau_{1}\right)$ and $\left[\tau_{2}^{\prime}, \tau_{4}\right)$ and so the evader is not under the attack of any pursuer on these intervals. Since $r(t)=1$ on $\left[\tau_{1}, \tau_{2}\right)$, therefore the evader is under the attack of the first pursuer on this interval, and so evader applies the manoeuvre $V_{1}(t)$ against the first pursuer on this interval. Also, we can see other manoeuvres of evader in formula (18).

The evader chooses his maneuvers nonanticipatively stage by stage as the game progresses. For the Example 3.5, the numbers $t_{1}, t_{2}, \ldots, t_{k} \in[0, T]$ in Definition 2.2 are defined as follows: $t_{0}=0, t_{1}=\tau_{1}, t_{2}=\tau_{2}, t_{3}=\tau_{3}, t_{4}=\tau_{3}^{\prime}, t_{5}=\tau_{2}^{\prime}, t_{6}=\tau_{4}$, $t_{7}=\tau_{5}, t_{8}=\tau_{5}^{\prime}, t_{9}=T$. As the time $t_{i}, i=1,2, \ldots, 8\left(t_{0}=0\right)$ occurs, the function $r(t)$ assigns the strategy $V_{r_{i}}(t)$ for the evader defined by (14), (15), where $r_{i}=r\left(t_{i}\right)$. The evader uses this strategy until $t_{i+1}$ occurs, that is, on the interval $t_{i} \leq t<t_{i+1}$. Note that $t_{i+1}$ is unspecified and defined as the first time when $r\left(t_{i}\right) \neq r\left(t_{i+1}\right)$.

In general, the numbers $t_{1}, t_{2}, \ldots, t_{k} \in[0, T]$ are defined as follows. By (14) the evader uses strategy $v(t)=V_{0}(t), \tau_{0} \leq t<\tau_{1}$. This means the evader applies $v(t)=V_{0}(t)$ until $\tau_{1}$ occurs. Set $t_{1}=\tau_{1}, t_{k}=T$. The numbers $t_{2}, \ldots, t_{k-1}$ are defined inductively. Let the time $t_{i} \in\left\{\tau_{1}, \tau_{1}^{\prime}, \ldots, \tau_{p}, \tau_{p}^{\prime}\right\}, i, p \geq 1$, occur and let $r_{i}=r\left(t_{i}\right)$. Then by (14) and (15) the evader applies the strategy $v(t)=V_{r_{i}}(t)$ starting from $t_{i}$ until the time $t_{i+1}$ occurs, for which $r\left(t_{i}\right) \neq r\left(t_{i+1}\right)$ for the first time, where $t_{i+1} \in\left\{\tau_{1}, \tau_{1}^{\prime}, \ldots, \tau_{p}, \tau_{p}^{\prime}, \tau_{p+1}, \tau_{p+1}^{\prime}\right\}$. As the time $t_{i+1}$ occurs the evader uses the strategy $v(t)=V_{r_{i+1}}(t)$ starting from the time $t_{i+1}$ where $r_{i+1}=r\left(t_{i+1}\right)$ and so on. To determine the times $\tau_{i}, i=1,2, \ldots, m_{0}$, the evader uses the current values of states $y_{i}(t), i=1,2, \ldots, m$. To this end, it suffices for the evader to know the current time $t$ and $x_{i}(t), i=1,2, \ldots, m$. Also, we can see from (14) and (15) that the strategy of evader has the form $v(t)=V_{r_{i}}(t)$ on the intervals $t_{i} \leq t<t_{i+1}, i=0,1, \ldots, k$. 
We now show that the strategy defined by the equations (14)-(17) is admissible. Indeed, let

$$
\begin{aligned}
f(t)= \begin{cases}(0, \alpha), & t \in[0, T) \backslash I_{1} \\
(\alpha, \alpha), & t \in I_{1} \\
(0,0), & t>T\end{cases} \\
g(t)= \begin{cases}\left(0,\left(\sum_{j=1}^{m}\left|u_{j}(t)\right|^{2}\right)^{1 / 2}\right), & t \in[0, T] \backslash I_{1}, \\
\left(\left|u_{r 1}(t)\right|,\left(\sum_{j=1}^{m} u_{j 2}^{2}(t)\right)^{1 / 2}\right), & t \in I_{1}, \\
\left(0,\left(\sum_{j=1}^{m}\left|u_{j}(t)\right|^{2}\right)^{1 / 2}\right), & t>T .\end{cases}
\end{aligned}
$$

Note that

$$
\int_{0}^{\infty}|f(s)|^{2} d s \leq 2 \alpha^{2} T, \quad|g(t)|^{2} \leq \sum_{j=1}^{m}\left|u_{j}(t)\right|^{2}
$$

Clearly, for $v(t)$ defined by (14)-(17) we have $v_{1}^{2}(t)+v_{2}^{2}(t)=|f(t)+g(t)|^{2}$. Therefore, using the Minkowskii inequality and (19) we obtain

$$
\begin{aligned}
\left(\int_{0}^{\infty}|v(s)|^{2} d s\right)^{1 / 2} & =\left(\int_{0}^{\infty}|f(s)+g(s)|^{2} d s\right)^{1 / 2} \\
& \leq\left(\int_{0}^{\infty}|f(s)|^{2} d s\right)^{1 / 2}+\left(\int_{0}^{\infty}|g(s)|^{2} d s\right)^{1 / 2} \\
& \leq\left(2 \alpha^{2} T\right)^{1 / 2}+\left(\int_{0}^{\infty} \sum_{j=1}^{m}\left|u_{j}(s)\right|^{2} d s\right)^{1 / 2} \\
& \leq \alpha \sqrt{2 T}+\left(\sum_{j=1}^{m} \rho_{i}^{2}\right)^{1 / 2}=\alpha \sqrt{2 T}+\rho \leq \sigma
\end{aligned}
$$

since by definition of $T, T_{0}$ and $\alpha$

$$
\begin{aligned}
\alpha \sqrt{2 T} & =\alpha \sqrt{2\left(T_{0}+\frac{2 a_{1}}{\alpha}\right)}=\sqrt{2 \alpha\left(\max _{i=1, \ldots, m}\left|x_{i 0}\right|+2 a_{1}\right)} \\
& \leq \sqrt{2 \alpha\left(\max _{i=1, \ldots, m}\left|x_{i 0}\right|+1\right)} \leq \sigma-\rho .
\end{aligned}
$$

Here, in the last inequality we used (7). Thus, the evasion strategy (14)-(17) is admissible.

Next, we prove the following statement. 
Lemma 3.6 The following are true

1) For all $i=1, \ldots, m$, we have $(i) y_{i 2}(t)>0$ for $t \geq T_{0}$ and (ii) $\tau_{i}^{\prime} \leq T$.

2) (i) if $x_{j 2}^{0}<0$ for some $j \in\{1, \ldots, m\}$, then $y_{j 2}(\theta)=0$ at some unique $\theta$, $0<\theta<T_{0}$, and $y_{j 2}(t)>0$ for all $t>\theta$.

(ii) if $x_{j 2}^{0} \geq 0$ for some $j \in\{1, \ldots, m\}$, then $y_{j 2}(t)>0$ for all $t>0$.

Proof We first show that $y_{i 2}\left(T_{0}\right)>0$ for all $i=1, \ldots, m$. Indeed, by (14)-(15) we have

$$
v_{2}(t) \geq \alpha+\left|u_{i 2}(t)\right|, 0 \leq t \leq T_{0},
$$

and therefore,

$$
\dot{y}_{i 2}(t)=e^{\lambda_{i} t}\left(v_{2}(t)-u_{i 2}(t)\right) \geq \alpha e^{\lambda_{i} t}>0 .
$$

Hence, $y_{i 2}(t), 0 \leq t \leq T_{0}$, increases strictly. By (21) we have

$$
\begin{aligned}
y_{i 2}\left(T_{0}\right) & =x_{i 2}^{0}+\int_{0}^{T_{0}} e^{\lambda_{i} s}\left(v_{2}(s)-u_{i 2}(s)\right) d s \\
& \geq x_{i 2}^{0}+\alpha \int_{0}^{T_{0}} e^{\lambda_{i} s} d s \geq x_{i 2}^{0}+\frac{\alpha}{\lambda_{i}}\left(e^{\lambda_{i} T_{0}}-1\right) \\
& >x_{i 2}^{0}+\frac{\alpha}{\lambda_{i}} \lambda_{i} T_{0} \geq-\left|x_{i}^{0}\right|+\max _{1 \leq j \leq m}\left|x_{j}^{0}\right| \geq 0 .
\end{aligned}
$$

Thus, $y_{i 2}\left(T_{0}\right)>0$ for all $i=1, \ldots, m$. Since $v_{2}(t) \geq\left|u_{i}(t)\right| \geq\left|u_{i 2}(t)\right|$ for $t>T_{0}$, therefore for $t>T_{0}$ we have

$$
\begin{aligned}
y_{i 2}(t) & =y_{i 2}\left(T_{0}\right)+\int_{T_{0}}^{t} e^{\lambda_{i} s}\left(v_{2}(s)-u_{i 2}(s)\right) d s \\
& \geq y_{i 2}\left(T_{0}\right)+\int_{T_{0}}^{t} e^{\lambda_{i} s}\left(\left|u_{i 2}(s)\right|-u_{i 2}(s)\right) d s \geq y_{i 2}\left(T_{0}\right)>0 .
\end{aligned}
$$

Thus, $y_{i 2}(t)>0$, for all $t \geq T_{0}$ and $i=1, \ldots, m$. In particular, we obtain that there is no $a_{k}$-approach time $\tau_{k}$ in the time interval $\left[T_{0}, \infty\right)$, since by definition (10) of an $a_{k}$-approach time $\tau_{k}$ one has to have $y_{k 2}\left(\tau_{k}\right)<0$. This is impossible for $\tau_{k} \geq T_{0}$ since by item 1) (i) $y_{k 2}(t)>0$ for all $t \geq T_{0}$. Thus, $\tau_{i} \leq T_{0}$ for all $i=1, \ldots, m_{0}$.

Next, by item 1) (ii) of Property 3.3 we have

$$
\tau_{i}^{\prime} \leq \tau_{i}+\frac{2 a_{i}}{\alpha} \leq T_{0}+\frac{2 a_{1}}{\alpha}=T,
$$

and the proof of item 1) of Lemma 3.6 follows. In particular, (22) implies that $I_{1} \subset$ $[0, T]$.

Remark 3.7 Due to the inclusion $I_{1} \subset[0, T]$ the set $[0, T] \cap I_{1}$ in (15) is equal to $I_{1}$. 


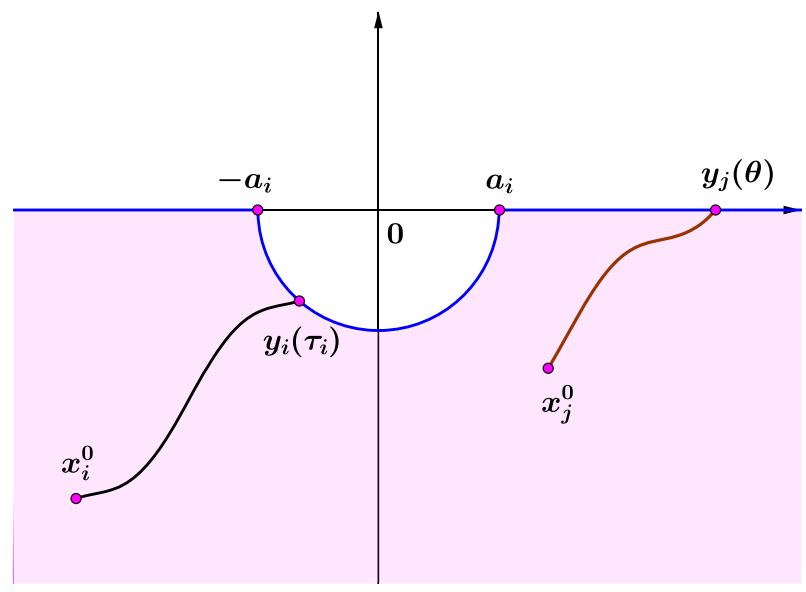

Fig. 2 Initial states have negative $y$-coordinates

Next, we prove item 2) (i). Since by (21) $y_{j 2}(t), 0 \leq t \leq T_{0}$, increases strictly and as shown above $y_{j 2}\left(T_{0}\right)>0$, then we necessarily have that $y_{j 2}(\theta)=0$ at some unique $\theta, 0<\theta<T_{0}$. In view of (20) we then obtain for $t>\theta$ that

$$
\begin{aligned}
y_{j 2}(t) & =y_{j 2}(\theta)+\int_{\theta}^{t} e^{\lambda_{j} s}\left(v_{2}(s)-u_{j 2}(s)\right) d s \\
& \geq \int_{[\theta, t] \cap\left[\theta, T_{0}\right]} e^{\lambda_{j} s}\left(\alpha+\left|u_{j 2}(s)\right|-u_{j 2}(s)\right) d s>0,
\end{aligned}
$$

which is the desired result. To show item 2) (ii), using $x_{j 2}^{0} \geq 0$ we observe that for $t \geq 0$,

$$
\begin{aligned}
y_{j 2}(t) & =x_{j 2}^{0}+\int_{0}^{t} e^{\lambda_{j} s}\left(v_{2}(s)-u_{j 2}(s)\right) d s \\
& \geq \int_{[0, t] \cap\left[0, T_{0}\right]} e^{\lambda_{j} s}\left(\alpha+\left|u_{j 2}(s)\right|-u_{j 2}(s)\right) d s>0 .
\end{aligned}
$$

This completes the proof of Lemma 3.6.

Item 1) (i) of Lemma 3.6 implies, in particular, that for the point $y_{j}$ with initial state $x_{j}^{0}$ for which $x_{j 2}^{0}<0$, the inequality $y_{j 2}\left(T_{0}\right)>0$ is satisfied. Thus, we necessarily have either $\left|y_{j}\left(\tau_{j}\right)\right|=a_{j}$ and $y_{j 2}\left(\tau_{j}\right)<0$ at some $0<\tau_{j}<T_{0}$ (see the point $y_{i}$ in Fig. 2) or $y_{j 2}(\theta)=0$ and $\left|y_{j 1}(\theta)\right| \geq a_{j}$ at some $0<\theta<T_{0}$ (see the point $y_{j}$ in Fig. 2). The former case will be studied in the following subsections in detail. In the latter case, we ignore the point $y_{j}(t)$ starting from the time $\theta$ since by Lemma 3.62 ) (i) we have $y_{j 2}(t)>0$ and so $y_{j}(t) \neq 0$ for all $t>\theta$. That is why in definition of 


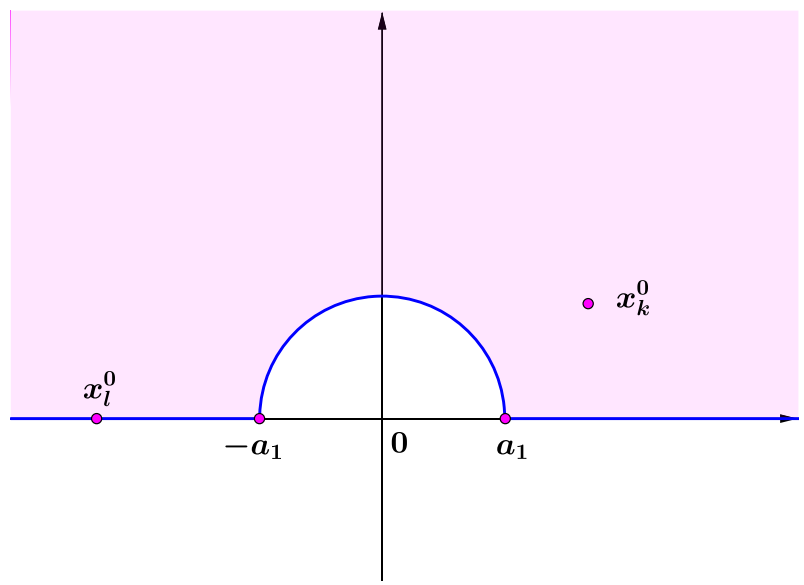

Fig. 3 Initial states have non negative $y$-coordinates

$a_{i}$-approach time $\tau_{i}(10)$ we required the inequality $y_{j 2}\left(\tau_{i}\right)<0$. The initial states $x_{k}^{0}$ with $x_{k 2}^{0}>0$, and $x_{l}^{0}$ with $x_{l 2}^{0}=0$, are shown in Fig. 3 . We ignore the corresponding points $y_{k}(t)$ and $y_{l}(t)$ as well for $t \geq 0$ since by Lemma 3.62 ) (ii) $y_{k 2}(t)>0$ and $y_{l 2}(t)>0$ for all $t \geq 0$.

Property 3.8 For any $i \in\left\{1, \ldots, m_{0}\right\}$ and $p \in\{1, \ldots, m\}$,

$$
\int_{\tau_{i}}^{\tau_{i}^{\prime}} e^{\lambda p^{s}} d s \leq e^{\Lambda T} \cdot \frac{4 a_{i}}{\alpha} .
$$

Proof We use (12) and $\tau_{i} \leq T_{0}<T$ to obtain

$$
\begin{aligned}
\int_{\tau_{i}}^{\tau_{i}^{\prime}} e^{\lambda_{p} s} d s & =\frac{1}{\lambda_{p}}\left(e^{\lambda_{p} \tau_{i}^{\prime}}-e^{\lambda_{p} \tau_{i}}\right) \\
& =\frac{e^{\lambda_{p} \tau_{i}}}{\lambda_{p}}\left(e^{\lambda_{p}\left(\tau_{i}^{\prime}-\tau_{i}\right)}-1\right) \leq \frac{1}{\lambda_{p}} e^{\Lambda T}\left(e^{\frac{2 \lambda_{p} a_{i}}{\alpha}}-1\right) .
\end{aligned}
$$

Since by (8) $\frac{2 \lambda_{p} a_{i}}{\alpha} \leq \frac{2 \Lambda a_{1}}{\alpha} \leq 1$, then using the inequality $e^{x}-1 \leq 2 x, 0 \leq x \leq 1$, in (24) we obtain (23). This completes the proof of the property.

\subsection{Auxiliary point $z_{p}$}

Take any $p \in\left\{1, \ldots, m_{0}\right\}$ and estimate $\left|y_{p}(t)\right|$ on $\left[\tau_{p}, \tau_{p}^{\prime}\right]$ assuming that $a_{p}$-approach was occurred at time $\tau_{p}$ with the point $y_{p}$. To this end we introduce an auxiliary point 
$z_{p}$ whose dynamics is described by the following equation

$$
z_{p}(t)=y_{p}\left(\tau_{p}\right)+\int_{\tau_{p}}^{t} e^{\lambda_{p} s}\left(V_{p}(s)-u_{p}(s)\right) d s, \tau_{p} \leq t \leq \tau_{p}^{\prime} .
$$

Note that the point $z_{p}(t)$ is defined only on the interval $\left[\tau_{p}, \tau_{p}^{\prime}\right]$. Since by $(15) v_{2}(t)=$ $U(t)$, therefore

$$
\begin{aligned}
z_{p 2}(t) & =z_{p 2}\left(\tau_{p}\right)+\int_{\tau_{p}}^{t} e^{\lambda_{p} s}\left(U(s)-u_{p 2}(s)\right) d s \\
& =y_{p 2}\left(\tau_{p}\right)+\int_{\tau_{p}}^{t} e^{\lambda_{p} s}\left(v_{2}(s)-u_{p 2}(s)\right) d s=y_{p 2}(t), \tau_{p} \leq t \leq \tau_{p}^{\prime} .
\end{aligned}
$$

Next, we show that

$$
\int_{\tau_{p}}^{\tau_{p}^{\prime}}\left|V_{p}(s)\right|^{2} d s \leq \sigma^{2}
$$

Indeed, denoting

$$
f_{1}(t)=(\alpha, \alpha), \quad g_{1}(t)=\left(\left|u_{p 1}(t)\right|,\left(\sum_{j=1}^{m} u_{j 2}(t)^{2}\right)^{1 / 2}\right)
$$

we obtain

$$
\begin{aligned}
\left|V_{p}(t)\right|^{2}=V_{p 1}^{2}(t)+U^{2}(t) & =\left(\alpha+\left|u_{p 1}(t)\right|\right)^{2}+\left(\alpha+\left(\sum_{j=1}^{m} u_{j 2}^{2}(t)\right)^{1 / 2}\right)^{2} \\
& =\left|f_{1}(t)+g_{1}(t)\right|^{2}
\end{aligned}
$$

Therefore, using the Minkowskii inequality and then item (1) of Property 3.3 we obtain

$$
\begin{aligned}
\left(\int_{\tau_{p}}^{\tau_{p}^{\prime}}\left|V_{p}(s)\right|^{2} d s\right)^{1 / 2} & =\left(\int_{\tau_{p}}^{\tau_{p}^{\prime}}\left|f_{1}(s)+g_{1}(s)\right|^{2} d s\right)^{1 / 2} \\
& \leq\left(\int_{\tau_{p}}^{\tau_{p}^{\prime}}\left|f_{1}(s)\right|^{2} d s\right)^{1 / 2}+\left(\int_{\tau_{p}}^{\tau_{p}^{\prime}}\left|g_{1}(s)\right|^{2} d s\right)^{1 / 2} \\
& \leq\left(2 \alpha^{2}\left(\tau_{p}^{\prime}-\tau_{p}\right)\right)^{1 / 2}+\left(\int_{\tau_{p}}^{\tau_{p}^{\prime}} \sum_{j=1}^{m}\left|u_{j}(s)\right|^{2} d s\right)^{1 / 2} \\
& \leq 2 \sqrt{\alpha a_{p}}+\rho<\sigma,
\end{aligned}
$$


since by (8) $a_{p} \leq a_{1}<\frac{(\sigma-\rho)^{2}}{4 \alpha}$, and hence (27) is true.

\subsection{Estimation of $\left|z_{p}(t)\right|$}

Let $\tau_{p} \leq t<\tau_{p}^{\prime}$ and for definiteness assume that $y_{p 1}\left(\tau_{p}\right) \geq 0$. Then by (16) we have $V_{p 1}(t)=\alpha+\left|u_{p 1}(t)\right|$. Therefore,

$$
\begin{aligned}
\left|z_{p}(t)\right| \geq z_{p 1}(t) & =y_{p 1}\left(\tau_{p}\right)+\int_{\tau_{p}}^{t} e^{\lambda_{p} s}\left(V_{p 1}(t)-u_{p 1}(t)\right) d s \\
& \geq \int_{\tau_{p}}^{t} e^{\lambda p_{p} s}\left(\alpha+\left|u_{p 1}(t)\right|-u_{p 1}(t)\right) d s \\
& \geq \alpha \int_{\tau_{p}}^{t} e^{\lambda_{p} s} d s=\frac{\alpha}{\lambda_{p}}\left(e^{\lambda_{p} t}-e^{\lambda_{p} \tau_{p}}\right) .
\end{aligned}
$$

On the other hand,

$$
\left|z_{p}(t)\right| \geq\left|y_{p}\left(\tau_{p}\right)\right|-\int_{\tau_{p}}^{t} e^{\lambda_{p} s}\left|V_{p}(s)-u_{p}(s)\right| d s .
$$

The integral in (29) can be estimated by using the Cauchy-Schwartz inequality as follows

$$
\begin{aligned}
\int_{\tau_{p}}^{t} e^{\lambda_{p} s}\left|V_{p}(s)-u_{p}(s)\right| d s & \leq\left(\int_{\tau_{p}}^{t} e^{2 \lambda_{p} s} d s\right)^{1 / 2}\left(\int_{\tau_{p}}^{t}\left|V_{p}(s)-u_{p}(s)\right|^{2} d s\right)^{1 / 2} \\
& \leq\left(\int_{\tau_{p}}^{t} e^{2 \lambda_{p} s} d s\right)^{1 / 2}\left(\int_{\tau_{p}}^{t} 2\left(\left|V_{p}(s)\right|^{2}+\left|u_{p}(s)\right|^{2}\right) d s\right)^{1 / 2} .
\end{aligned}
$$

Since by (27) and the admissibility of control $u_{p}(s)$

$$
\int_{\tau_{p}}^{t}\left|V_{p}(s)\right|^{2} d s \leq \sigma^{2}, \quad \int_{\tau_{p}}^{t}\left|u_{p}(s)\right|^{2} d s \leq \rho_{p}^{2} \leq \sigma^{2},
$$

then it follows from (30) that

$$
\int_{\tau_{p}}^{t} e^{\lambda_{p} s}\left|V_{p}(s)-u_{p}(s)\right| d s \leq 2 \sigma\left(\int_{\tau_{p}}^{t} e^{2 \lambda_{p} s} d s\right)^{1 / 2}
$$

Since by (22) $\tau_{p} \leq t \leq \tau_{p}^{\prime} \leq T$, therefore

$$
\int_{\tau_{p}}^{t} e^{2 \lambda_{p} s} d s=\frac{1}{2 \lambda_{p}}\left(e^{\lambda_{p} t}+e^{\lambda_{p} \tau_{p}}\right)\left(e^{\lambda_{p} t}-e^{\lambda_{p} \tau_{p}}\right) \leq \frac{e^{\Lambda T}}{\lambda_{p}}\left(e^{\lambda_{p} t}-e^{\lambda_{p} \tau_{p}}\right) .
$$


Then by (31) we can see that

$$
\int_{\tau_{p}}^{t} e^{\lambda_{p} s}\left|V_{p}(s)-u_{p}(s)\right| d s \leq 2 \sigma \sqrt{\frac{e^{\Lambda T}}{\lambda_{p}}\left(e^{\lambda_{p} t}-e^{\lambda_{p} \tau_{p}}\right)} .
$$

Combining (29) and (32), and using the equation $\left|y_{p}\left(\tau_{p}\right)\right|=a_{p}$ yields that

$$
\left|z_{p}(t)\right| \geq a_{p}-2 \sigma \sqrt{\frac{e^{\Lambda T}}{\lambda_{p}}\left(e^{\lambda_{p} t}-e^{\lambda_{p} \tau_{p}}\right)} .
$$

It is easily seen from (28) and (33) that

$$
\left|z_{p}(t)\right| \geq f(t)=\max \left\{f_{1}(t), f_{2}(t)\right\}, \quad t \geq \tau_{p},
$$

where

$$
f_{1}(t)=\frac{\alpha}{\lambda_{p}}\left(e^{\lambda_{p} t}-e^{\lambda_{p} \tau_{p}}\right), \quad f_{2}(t)=a_{p}-2 \sigma \sqrt{\frac{e^{\Lambda T}}{\lambda_{p}}\left(e^{\lambda_{p} t}-e^{\lambda_{p} \tau_{p}}\right)}
$$

Note that $f_{1}(t), t \geq \tau_{p}$, increases from 0 to $\infty$ and $f_{2}(t), t \geq \tau_{p}$, decreases from $a_{p}$ to $-\infty$. It is not difficult to see that $f(t), t \geq \tau_{p}$, attains its minimum at the point $t=t_{*}$, where

$$
f_{1}(t)=f_{2}(t), \quad t \geq \tau_{p} .
$$

Let $\left(e^{\lambda_{p} t}-e^{\lambda_{p} \tau_{p}}\right)^{1 / 2}=z$. Then Eq. (35) takes the form

$$
\frac{\alpha}{\lambda_{p}} z^{2}=a_{p}-2 \sigma \sqrt{\frac{e^{\Lambda T}}{\lambda_{p}}} z
$$

or $\alpha z^{2}+2 \sigma \sqrt{e^{\Lambda T} \lambda_{p}} z-a_{p} \lambda_{p}=0$. This equation has the following positive root

$$
\begin{aligned}
z_{*} & =\frac{\sqrt{\lambda_{p}}}{\alpha}\left(-\sigma \sqrt{e^{\Lambda T}}+\sqrt{\sigma^{2} e^{\Lambda T}+a_{p} \alpha}\right) \\
& =\frac{a_{p} \sqrt{\lambda_{p}}}{\sigma \sqrt{e^{\Lambda T}}+\sqrt{\sigma^{2} e^{\Lambda T}+a_{p} \alpha}} .
\end{aligned}
$$

Then

$$
\min _{t \geq \tau_{p}} f(t)=f\left(t_{*}\right)=f_{1}\left(t_{*}\right)=\frac{\alpha}{\lambda_{p}} z_{*}^{2}=\frac{\alpha}{\lambda_{p}} \cdot \frac{a_{p}^{2} \lambda_{p}}{\left(\sigma \sqrt{e^{\Lambda T}}+\sqrt{\sigma^{2} e^{\Lambda T}+a_{p} \alpha}\right)^{2}} .
$$


By (8) we have $a_{1}<\frac{\sigma^{2}}{32 \alpha}<\frac{1}{\alpha} \sigma^{2} e^{\Lambda T}$, and so $a_{p} \alpha \leq a_{1} \alpha<\sigma^{2} e^{\Lambda T}$, therefore (36) implies that

$$
\left|z_{p}(t)\right| \geq \min _{t \geq \tau_{p}} f(t)>\frac{\alpha a_{p}^{2}}{6 \sigma^{2} e^{\Lambda T}}
$$

Next, since by definition

$$
\tau_{p}^{\prime}=\frac{1}{\lambda_{p}} \ln \left(e^{\lambda_{p} \tau_{p}}+\frac{2 \lambda_{p} a_{p}}{\alpha}\right)
$$

using the fact that $y_{p 2}\left(\tau_{p}\right) \geq-\left|y_{p}\left(\tau_{p}\right)\right|=-a_{p}$ we obtain

$$
\begin{aligned}
z_{p 2}\left(\tau_{p}^{\prime}\right) & =y_{p 2}\left(\tau_{p}\right)+\int_{\tau_{p}}^{\tau_{p}^{\prime}} e^{\lambda_{p} s}\left(U(s)-u_{p 2}(s)\right) d s \\
& \geq-a_{p}+\int_{\tau_{p}}^{\tau_{p}^{\prime}} e^{\lambda_{p} s}\left(\alpha+\left(\sum_{i=1}^{m} u_{i 2}(s)^{2}\right)^{1 / 2}-u_{p 2}(s)\right) d s \\
& \geq-a_{p}+\alpha \int_{\tau_{p}}^{\tau_{p}^{\prime}} e^{\lambda_{p} s} d s=-a_{p}+\frac{\alpha}{\lambda_{p}}\left(e^{\lambda_{p} \tau_{p}^{\prime}}-e^{\lambda_{p} \tau_{p}}\right) \\
& =-a_{p}+\frac{\alpha}{\lambda_{p}}\left(e^{\lambda_{p} \tau_{p}}+\frac{2 \lambda_{p} a_{p}}{\alpha}-e^{\lambda_{p} \tau_{p}}\right)=a_{p} .
\end{aligned}
$$

Finally, let $t \geq \tau_{p}^{\prime}$. By (26) $y_{p 2}\left(\tau_{p}^{\prime}\right)=z_{p 2}\left(\tau_{p}^{\prime}\right)$, and by (14), (15) and (17), $v_{2}(t) \geq$ $\left|u_{p 2}(t)\right|$. Then using (17), (38) we get

$$
y_{p 2}(t)=z_{p 2}\left(\tau_{p}^{\prime}\right)+\int_{\tau_{p}^{\prime}}^{t} e^{\lambda_{p} s}\left(v_{2}(s)-u_{p 2}(s)\right) d s \geq z_{p 2}\left(\tau_{p}^{\prime}\right) \geq a_{p} .
$$

Thus, we have the following inequalities

$$
\begin{aligned}
& \left|z_{p}(t)\right|>\frac{\alpha a_{p}^{2}}{6 \sigma^{2} e^{\Lambda T}}, \quad \tau_{p} \leq t \leq \tau_{p}^{\prime}, \\
& y_{p 2}(t) \geq a_{p}, \quad t \geq \tau_{p}^{\prime} .
\end{aligned}
$$

\subsection{Estimation of $\left|y_{p}(t)-z_{p}(t)\right|$}

We have

$$
\left|y_{p}(t)-z_{p}(t)\right|=\left|\int_{\tau_{p}}^{t}\left(v(s)-V_{p}(s)\right) d s\right|, \quad \tau_{p} \leq t \leq \tau_{p}^{\prime} .
$$


By (15) and (26)

$$
v(t)=\left(V_{r 1}(t), U(t)\right), \quad V_{p}(t)=\left(V_{p 1}(t), U(t)\right), \quad \tau_{p} \leq t<\tau_{p}^{\prime} .
$$

Consider two cases: (i) $\tau_{p}^{\prime} \leq \tau_{p+1}$ and (ii) $\tau_{p+1} \leq \tau_{p}^{\prime}$.

Case (i). Let $\tau_{p}^{\prime} \leq \tau_{p+1}$. Then by item (1) of Property $3.4 r=r(t)=p$ for $\tau_{p} \leq t<\tau_{p}^{\prime}$. Therefore by (42) we have $v(t)=V_{p}(t), \tau_{p} \leq t<\tau_{p}^{\prime}$. Hence, by (41)

$$
\left|y_{p}(t)-z_{p}(t)\right|=0
$$

Case (ii). Assume now $\tau_{p+1} \leq \tau_{p}^{\prime}$. Then by item (2) of Property 3.4 we have $v(t)=\left(V_{p 1}(t), U(t)\right), \tau_{p} \leq t<\tau_{p+1}$. Therefore, (41) leads to

$$
\begin{aligned}
& \left|y_{p}(t)-z_{p}(t)\right| \\
& =\left|\int_{\tau_{p+1}}^{t} e^{\lambda_{p} s}\left(v(s)-V_{p}(s)\right) d s\right| \leq \int_{\tau_{p+1}}^{t} e^{\lambda_{p} s}\left|v(s)-V_{p}(s)\right| d s \\
& \leq \int_{\left[\tau_{p+1}, t\right) \backslash I_{p+1}} e^{\lambda p s}\left|v(s)-V_{p}(s)\right| d s+\int_{\left[\tau_{p+1}, t\right) \cap I_{p+1}} e^{\lambda_{p} s}\left|v(s)-V_{p}(s)\right| d s .(44)
\end{aligned}
$$

Since by definition $r(t)=p, t \in\left[\tau_{p}, \tau_{p}^{\prime}\right) \backslash I_{p+1}$ and $\left[\tau_{p+1}, t\right) \backslash I_{p+1} \subset\left[\tau_{p}, \tau_{p}^{\prime}\right) \backslash I_{p+1}$, therefore we have $r=r(t)=p$, and hence, $v(t)=V_{p}(t)$ for $t \in\left[\tau_{p+1}, t\right) \backslash I_{p+1}$. Consequently, the first integral in (44) is 0 , and so (44) takes the form

$$
\left|y_{p}(t)-z_{p}(t)\right| \leq \int_{\left[\tau_{p+1}, t\right] \cap I_{p+1}} e^{\lambda_{p} s}\left|v(s)-V_{p}(s)\right| d s .
$$

By (16) and (42)

$$
\left|v(s)-V_{p}(s)\right|=\left|V_{r 1}(s)-V_{p 1}(s)\right| \leq 2 \alpha+\left|u_{r 1}(s)\right|+\left|u_{p 1}(s)\right|,
$$

and therefore (45) implies that

$$
\left|y_{p}(t)-z_{p}(t)\right| \leq \int_{I_{p+1}} e^{\lambda p s}\left(2 \alpha+\left|u_{r 1}(s)\right|+\left|u_{p 1}(s)\right|\right) d s .
$$

To estimate the integral in (46), we need to estimate the integrals

$$
\int_{I_{p+1}} e^{\lambda_{p} s} d s, \int_{I_{p+1}} e^{\lambda_{p} s}\left|u_{r 1}(s)\right| d s, \text { and } \int_{I_{p+1}} e^{\lambda_{p} s}\left|u_{p 1}(s)\right| d s .
$$

The first integral can be estimated using (23) and Property 3.2 as follows

$$
\int_{I_{p+1}} e^{\lambda_{p} s} d s \leq \sum_{i=p+1}^{m} \int_{\tau_{i}}^{\tau_{i}^{\prime}} e^{\lambda_{p} s} d s \leq \sum_{i=p+1}^{m} e^{\Lambda T} \frac{4 a_{i}}{\alpha} \leq e^{\Lambda T} \frac{8 a_{p+1}}{\alpha} .
$$


Next, we estimate the second integral in (47). Using the Cauchy-Schwartz inequality we have

$$
\int_{I_{p+1}} e^{\lambda_{p} s}\left|u_{p 1}(s)\right| d s \leq\left(\int_{I_{p+1}} e^{2 \lambda_{p} s} d s\right)^{1 / 2} \cdot\left(\int_{I_{p+1}}\left|u_{p 1}(s)\right|^{2} d s\right)^{1 / 2} .
$$

Since

$$
\int_{I_{p+1}}\left|u_{p 1}(s)\right|^{2} d s \leq \sum_{i=1}^{m} \int_{0}^{\infty}\left|u_{i}(s)\right|^{2} d s \leq \sigma^{2}
$$

and similar to (48) we get

$$
\int_{I_{p+1}} e^{2 \lambda_{p} s} d s \leq \sum_{i=p+1}^{m} \int_{\tau_{i}}^{\tau_{i}^{\prime}} e^{2 \lambda_{p} s} d s \leq e^{2 \Lambda T} \frac{8 a_{p+1}}{\alpha}
$$

Then it follows from (49) that

$$
\int_{I_{p+1}} e^{\lambda_{p} s}\left|u_{p 1}(s)\right| d s \leq \sigma e^{\Lambda T} \sqrt{\frac{8 a_{p+1}}{\alpha}}
$$

Similarly, for the third integral in (47), we have

$$
\int_{I_{p+1}} e^{\lambda_{p} s}\left|u_{p 1}(s)\right| d s \leq \sigma e^{\Lambda T} \sqrt{\frac{8 a_{p+1}}{\alpha}}
$$

Combining (48), (50), and (51) we obtain from (46) that

$$
\left|y_{p}(t)-z_{p}(t)\right| \leq 2 \alpha \cdot \frac{8 a_{p+1}}{\alpha} \cdot e^{\Lambda T}+2 \sigma e^{\Lambda T} \sqrt{\frac{8 a_{p+1}}{\alpha}} \leq 3 \sigma e^{\Lambda T} \sqrt{\frac{8 a_{p+1}}{\alpha}}
$$

using the inequality

$$
16 a_{p+1}<\sigma \sqrt{\frac{8 a_{p+1}}{\alpha}}
$$

which follows from the inequalities $a_{p+1} \leq a_{1}<\frac{\sigma^{2}}{32 \alpha}$ (see (8)).

Thus,

$$
\left|y_{p}(t)-z_{p}(t)\right| \leq 3 \sigma e^{\Lambda T} \sqrt{\frac{8 a_{p+1}}{\alpha}}, \tau_{p} \leq t \leq \tau_{p}^{\prime} .
$$




\subsection{Estimation of $\left|y_{p}(t)\right|$}

Using (39) and (52) we obtain

$$
\left|y_{p}(t)\right| \geq\left|z_{p}(t)\right|-\left|y_{p}(t)-z_{p}(t)\right| \geq \frac{\alpha a_{p}^{2}}{6 \sigma^{2} e^{\Lambda T}}-3 \sigma e^{\Lambda T} \sqrt{\frac{8 a_{p+1}}{\alpha}} \geq \frac{\alpha a_{p}^{2}}{12 \sigma^{2} e^{\Lambda T}},
$$

for $t \in\left[\tau_{p}, \tau_{p}^{\prime}\right]$ since by (9)

$$
a_{p+1} \leq \frac{\alpha^{3}}{8 \cdot 6^{4} \sigma^{6} e^{4 \Lambda T}} a_{p}^{4}
$$

Also, it follows from the definition of $\kappa$ and the inequality $a_{p}<1$ that

$$
a_{p+1} \leq \frac{\alpha}{16 \sigma^{2} e^{\Lambda T}} a_{p}^{4} \leq \frac{\alpha}{12 \sigma^{2} e^{\Lambda T}} a_{p}^{2}
$$

Therefore, (53) implies that $\left|y_{p}(t)\right|>a_{p+1}$. Also, by (40)

$$
y_{p 2}(t) \geq a_{p}, \quad t \geq \tau_{p}^{\prime} .
$$

Thus,

$$
\begin{aligned}
& \left|y_{p}(t)\right| \geq a_{p}, \text { for } 0 \leq t \leq \tau_{p}, \quad\left(\text { by definition of } \tau_{p}\right) \\
& \left|y_{p}(t)\right| \geq \frac{\alpha a_{p}^{2}}{12 \sigma^{2} e^{\Lambda T}}>a_{p+1}, \text { for } \tau_{p} \leq t \leq \tau_{p}^{\prime}, \quad(\text { by }(53)) \\
& y_{p 2}(t) \geq a_{p}, \text { for } t \geq \tau_{p}^{\prime}, \quad(\text { by }(40))
\end{aligned}
$$

Thus we conclude:

If an $a_{p}$-approach time $\tau_{p}$ occurs with the point $y_{p}$ (see the point $y_{i}$ in Fig. 2), then $y_{p}(t) \neq 0$, for all $t \geq 0$ (see (54)-(56)). Moreover, for any $i \geq p+1$, there is no $a_{i}$-approach time $\tau_{i}$ for the point $y_{p}$

(1) on the time interval $\left[\tau_{p}, \tau_{p}^{\prime}\right]$ since $\left|y_{p}(t)\right|>a_{p+1} \geq a_{i}$ for any $i \geq p+1$ (see (55)).

(2) on the time interval $\left[\tau_{p}^{\prime}, \infty\right)$, since $\left|y_{p}(t)\right| \geq y_{p 2}(t) \geq a_{p}>a_{i}$ for any $i \geq p+1$ (see (56)).

The proof of Theorem 3.1 is completed.

\section{Conclusion}

We have studied a linear evasion differential game of many pursuers and one evader. We have constructed a strategy for the evader and proved the possibility of evasion. The evader uses a manoeuvre on the set $I_{1}$ and on the set $[0, T] \backslash I_{1}$ evader uses the 
control $v(t)=\left(0, \alpha+\left(\sum_{j=1}^{m}\left|u_{j}(t)\right|^{2}\right)^{1 / 2}\right)$. The measure of the set $I_{1}$ can be made by choosing parameters $a_{1}$ and $\alpha$ as small as we wish. We have also shown that all the approach times $\tau_{i}$ can occur only before a specified time $T_{0}$, moreover $\tau_{i}^{\prime} \leq T$. The total number of approach times $\tau_{i}$ of all pursuers doesn't exceed the number of pursuers $m$. For $t \geq T$, the evader uses the control $v(t)=\left(0,\left(\sum_{j=1}^{m}\left|u_{j}(t)\right|^{2}\right)^{1 / 2}\right)$ and there is no longer an approach time occurs. The main contributions of the paper are (i) the construction of evasion strategy, (ii) estimating the distances of objects from the origin, (iii) the possibility of evasion from many pursuers.

Acknowledgements Open access funding provided by Università degli Studi Mediterranea di Reggio Calabria within the CRUI-CARE Agreement. We sincerely thank the anonymous reviewers for their careful reading of our manuscript and their many insightful comments and suggestions which helped us to improve the quality of the paper. This work was completed during the stay of the author Ibragimov G.I. at University Mediterranea of Reggio Calabria-Dept Di.Gi.ES—as Visiting Researcher and it was partially supported by Geran Putra Berimpak UPM/700-2/1/GPB/2017/9590200 of Universiti Putra Malaysia and the financial support by Decisions_LAB-Dept. of Law, Economics and Human Sciences-University Mediterranea of Reggio Calabria, Italy.

Open Access This article is licensed under a Creative Commons Attribution 4.0 International License, which permits use, sharing, adaptation, distribution and reproduction in any medium or format, as long as you give appropriate credit to the original author(s) and the source, provide a link to the Creative Commons licence, and indicate if changes were made. The images or other third party material in this article are included in the article's Creative Commons licence, unless indicated otherwise in a credit line to the material. If material is not included in the article's Creative Commons licence and your intended use is not permitted by statutory regulation or exceeds the permitted use, you will need to obtain permission directly from the copyright holder. To view a copy of this licence, visit http://creativecommons.org/licenses/by/4.0/.

\section{References}

Alexander S, Bishop R, Christ R (2009) Capture pursuit games on unbounded domain. Lënseignement Mathëmatique 55(1/2):103-125

Alias IA, Ibragimov G, Rakhmanov A (2016) Evasion differential game of infinitely many evaders from infinitely many pursuers in Hilbert space. Dyn Games Appl 6(2):1-13. https://doi.org/10.1007/s13235016-0196-0

Azamov AA (2008) A lower bound for the advantage coefficient in a search problem on graphs. Differ Equ 44(12): 1764-1767

Bakolas E, Tsiotras P (2011) On the relay pursuit of a maneuvering target by a group of pursuers. In: 50th IEEE conference on decision and control and European control conference, Orlando, FL, 2011, pp 4270-4275

Bannikov AS, Petrov NN (2010) To non-stationary group pursuit problem. Trudy Inst Math Mech UrO RAN 16(1):40-51

Başar T, Olsder G (1999) Dynamic noncooperative game theory. Reprint of the second (1995) edition. Classics in Applied Mathematics, 23. SIAM

Berkovitz LD (1986) Differential game of generalized pursuit and evasion. SIAM J Control 24(3):361-373

Bhattacharya S, Başar T, Hovakimyan N (2016) A visibility-based pursuit-evasion game with a circular obstacle. J Optim Theory Appl 171(3):1071-1082

Blagodatskikh AI, Petrov NN (2009) Conflict interaction between groups of controlled objects. Udmurt State University Press, Izhevsk (in Russian)

Borowko P, Rzymowski W, Stachura A (1988) Evasion from many pursuers in the simple motion case. J Math Anal Appl 135(1):75-80

Buckdahn R, Cardaliaguet P, Quincampoix M (2011) Some recent aspects of differential game theory. Dyn Games Appl 1(1):74-114 
Cardaliaguet P, Quincampoix M, Saint-Pierre P (2000) Pursuit differential games with state constraints. SIAM J Control Optim 39(5):1615-1632

Chernous'ko FL (1976) A problem of evasion of several pursuers. Prikl Mat Mekh 40(1):14-24

Chernous'ko FL, Zak VL (1985) On differential games of evasion from many pursuers. J Optim Theory Appl 46(4):461-470

Chikrii AA, Prokopovich PV (1992) Simple pursuit of one evader by a group. Cybern Syst Anal 28(3):438444. https://doi.org/10.1007/BF01125424

Chodun W (1989) Differential games of evasion with many pursuers. J Math Anal Appl 142(2):370-389

Croft HT (1964) Lion and man: a postscript. J Lond Math Soc 39:385-390

Friedman A (1971) Differential games. Wiley, New York

Grigorenko NL (1990) Mathematical methods of control of several dynamic processes. MSU Press, Moscow (in Russian)

Hajek O (1975) Pursuit games. Mathematical science engineering. Academic Press, New York

Hamilton W (1971) Geometry for the selfish herd. J Theor Biol 31(2):295-311

Ibragimov GI, Salimi M, Amini M (2012) Evasion from many pursuers in simple motion differential game with integral constraints. Eur J Oper Res 218(2):505-511

Ibragimov G, Ferrara M, Kuchkarov A, Pansera BA (2018) Simple motion evasion differential game of many pursuers and evaders with integral constraints. Dyn Games Appl 8:352-378. https://doi.org/10. 1007/s13235-017-0226-6

Isaacs R (1965) Differential games. Wiley, New York

Ivanov RP (1980) Simple pursuit-evasion on a compact convex set. Doklady Akademii Nauk SSSR 254(6):1318-1321

Konstantinidis G, Kehagias A (2016) Simultaneously moving cops and robbers. Theor Comput Sci 645:4859

Krasovskii NN, Subbotin AI (1988) Game-theoretical control problems. Springer, New York

Kuang FH (1986) Sufficient conditions of capture in differential games of $m$ pursuer and one evader. Kibernetika 6:91-97

Kuchkarov A, Ibragimov G, Ferrara M (2016) Simple motion pursuit and evasion differential games with many pursuers on manifolds with Euclidean metric. Discrete Dyn Nat Soc 2016:1386242. https://doi. org/10.1155/2016/1386242

Kumkov SS, Le Ménec S, Patsko VS (2017) Zero-sum pursuit-evasion differential games with many objects: survey of publications. Dyn Games Appl 7:609-633. https://doi.org/10.1007/s13235-016-0209-z

Mishchenko EF, Nikol'skii MS, Satimov NY (1977) Evoidance encounter problem in differential games of many persons. Trudy MIAN USSR 143:105-128

Petrosyan LA (1993) Differential games of pursuit. World Scientific, Singapore

Pontryagin LS (1988) Selected works. Nauka, Moscow

Pontryagin LS, Mischenko YF (1971) The problem of evasion in linear differential games. Differencial'nye Uravnenija 7(3):436-445

Pshenichnii BN (1976) Simple pursuit by several objects. Cybern Syst Anal 12(3):145-146

Pshenichnii BN, Chikrii AA, Rappoport JS (1981) An efficient method of solving differential games with many pursuers. Dokl Akad Nauk SSSR 256:530-535 (In Russian)

Scott WL, Leonard NE (2018) Optimal evasive strategies for multiple interacting agents with motion constraints. Autom J IFAC 94:26-34

Sh KA, Risman MH, Malik AH (2012) Differential games with many pursuers when evader moves on the surface of a cylinder. Anziam J. 53(E):E1-E20

Sun W, Tsiotras P (2014) An optimal evader strategy in a two-pursuer one-evader problem. In: Proceedings of 53rd IEEE conference decision and control, Los Angeles, CA, pp 4266-4271

Vagin D, Petrov N (2001) The problem of the pursuit of a group of rigidly coordinated evaders. J Comput Syst Sci Int 40(5):749-753

Zak VL (1978) On a problem of evading many pursuers. J Appl Math Mekhs 43(3):492-501

Zak VL (1981) Problem of evasion from many pursuers controlled by acceleration. Izvestiya Akademii Nauk SSSR Tekhnicheskaya Kibernetika 2:57-71

Zak VL (1982) The problem of evading several pursuers in the presence of a state constraint. Sov Math Dokl 26(1):190-193

Publisher's Note Springer Nature remains neutral with regard to jurisdictional claims in published maps and institutional affiliations. 